\title{
Optical Control of Couplings in Polariton Condensate Lattices
}

\author{
S. Alyatkin, ${ }^{1}$ J. D. Töpfer,${ }^{1,2}$ A. Askitopoulos ${ }^{1}$ H. Sigurdsson,,${ }^{1,2}$ and P. G. Lagoudakis ${ }^{1,2, *}$ \\ ${ }^{1}$ Skolkovo Institute of Science and Technology, Novaya St. 100, Skolkovo 143025, Russian Federation \\ ${ }^{2}$ School of Physics and Astronomy, University of Southampton, Southampton, SO17 1BJ, United Kingdom
}

(Dated: May 5, 2020)

\begin{abstract}
We demonstrate deterministic control of the nearest and next-nearest neighbor coupling in the unit cell of a square lattice of microcavity exciton-polariton condensates. We tune the coupling in a continuous and reversible manner by optically imprinting potential barriers of variable height, in the form of spatially localized incoherent exciton reservoirs that modify the particle flow between condensates. By controlling the couplings in a $2 \times 2$ polariton cluster, we realize ferromagnetic, anti-ferromagnetic and paired ferromagnetic phases, and demonstrate the potential scalability of the system.
\end{abstract}

Control over a novel type of a many-body optical network, the exciton-polariton condensate lattice, is highly desirable partly due to its application in integrated optical circuitry [1], potential for quantum computation $[2,3]$ as well as a testbed for the study of emergent phenomena in large complex systems; these range from the Kibble-Zurek mechanism [4], spontaneous magnetization [5], topological phases [6], to reverse ground state annealing [7]. Indeed, exciton-polaritons (from here on polaritons) have already found a role in various memory processing elements such as logic gates [8], transistors $[9,10]$, switches [11], routers [12], and diodes [13], ranging from cryogenic to room-temperature operation conditions. The accuracy with which one can deterministically control the interactions between individual polariton condensates reflects the experimental control with which one can design advanced polaritonic devices, and access interesting phases of interacting many-body systems.

Polariton condensates [14] are coherent ensembles of bosonic light-matter quasi-particles that in the mean field treatment, are described by a macroscopic wavefunction dictated by a complex nonlinear Schrödinger equation. Interactions between trapped polariton condensates can be accurately quantified using the tightbinding treatment, where the condensates can display synchronization [15], Josephson oscillations [16], frequency combs [17], and other intriguing effects stemming from their non-Hermitian and strong nonlinear character. Relevant to the current study, the interaction strength between spatially separated polariton condensates can be dramatically enhanced by realizing them not in a trapping geometry but instead as stable ballistically expanding optical fluids sustained by the gain of the excitation source [18-20]. Recently, a study on the interactions between expanding polariton condensates revealed that their dynamics are dictated by time-delayed equations of motion similar to the Lang-Kobayashi equation used to describe coupled laser systems [19, 21]. Therefore, designing a network of these expanding polariton condensates brings in a new platform for the study of nonlinear oscillatory systems used to describe chaos, neurological functions, social behavior, and synchronization [22]. On the other hand, inspired by recent developments on Ising machines based on optical parametric oscillators [23, 24], networks of polariton condensates can potentially be designed to tackle computationally challenging problems in an analogue manner by associating the degrees of freedom of the condensates (i.e., amplitude, phase, and polarization) to appropriate spin Hamiltonians [7] that can be mapped to computationally complex tasks [25]. Either case would require programmable coupling strengths between condensates. Recently, gates with dissipation inbetween condensate nodes were proposed for arbitrary interaction control in condensate lattices [26].

In this Letter, we demonstrate an all-optical method to tune and measure the coupling between adjacent polariton condensates. Different from lattices of polariton condensates realized in lithographically written structures [27], here we optically imprint networks of polariton condensates using tightly focused non-resonant optical beams in a planar microcavity shaped by a spatial light modulator (SLM). This allows for both precise and rewritable control of the excitation profile that makes it possible to drive condensate networks of various geometries in a microcavity. Furthermore, we additionally imprint optically incoherent exciton reservoirs of controllable density along the edges connecting polariton condensates. The repulsive interaction between reservoir excitons and condensate polaritons acts as a barrier that modifies the polariton flow between adjacent condensates $[9,28,29]$. We show that by changing the excitation power of these excitonic barriers, and subsequently their potential height, we can tune the phase of the polariton flow between condensates, altering their interference. We implement this concept to demonstrate a programmable $1 \times 2,2 \times 2$, and $4 \times 4$ polariton condensate clusters.

We use a strain compensated $2 \lambda$ GaAs based planar microcavity with embedded three pairs of $\operatorname{In}_{0.08} \mathrm{Ga}_{0.92} \mathrm{As}$ quantum wells described in Ref. [30]. The sample is held at $\approx 4 \mathrm{~K}$ within a closed-cycle helium cryostat and pumped non-resonantly using a continuous wave (CW) single mode laser at $1.5578 \mathrm{eV}$, at an exciton-photon 
detuning of $-3.8 \mathrm{meV}$. The polariton photoluminescence $(\mathrm{PL})$ is collected using a long-pass filter. The excitation profile is controlled with a reflective phase-only SLM which modulates the incident laser beam. We start with the simplest building block of the condensate lattice - a polariton dyad. Figure 1(a) shows the pump profile consisting of two laser spots that are tightly focused (FWHM $\approx 1.6 \mu \mathrm{m}$ ) with a microscope objective of $\mathrm{NA}=0.42$ and separated by a distance $d \approx 15.7 \mu \mathrm{m}$. Each of the two condensates is driven at a power of $1.3 \times \mathrm{P}_{\mathrm{thr}}$, where $\mathrm{P}_{\text {thr }}$ is the threshold power (corresponds to threshold intensity of $\approx 418 \mathrm{~kW} / \mathrm{cm}^{2}$ or $52 \mathrm{~kW} / \mathrm{cm}^{2}$ per quantum well) determined for a single isolated condensate, occurring at $1.4485 \mathrm{eV}$. The constructive interference in the real space PL at the center between the two condensates [Fig. 1(b)], and the bright central vertical fringe in the reciprocal space PL [white dashed line in Fig. 1(c)] indicate in-phase locking of the dyad [20]. Here, we denote in-phase and anti-phase configuration with parallel and anti-parallel white arrows (spins) resembling ferromagnetic (FM) and anti-ferromagnetic (AFM) type of arrangement of phases between the condensates. Continuous tuning of the separation distance in a polariton dyad reveals parity-flip transitions [19]. In this study, we tune to a dyad separation distance resulting in a stationary phase configuration.

Next, we investigate the effect of an optically induced barrier on the coupling of the dyad. A third laser beam injects non-resonantly an exciton reservoir in the middle of the dyad. We use cross-circularly polarized excitation for the barrier with respect to the condensates pumps in order to minimize gain due to overlap of the condensate wavefunctions with the barrier. The cross-circular configuration allows for a wider tunability of the barrier exciton reservoir density before barrier-induced nonlinearities set in, i.e. before condensation occurs at the barrier. Figure $1(\mathrm{~d})$ shows the pumping profile for a polariton dyad in the presence of a weak barrier pumped below threshold with $\mathrm{P}_{\text {bar }}=0.36 \times \mathrm{P}_{\text {thr }}$. The resulting interference patterns of the polariton $\mathrm{PL}$ in real space [Fig. 1(e)] and reciprocal space $[$ Fig. $1(f)]$ reveal that the phase configuration in the presence of the barrier has switched from FM to AFM. We note that the barrier injects a negligible amount of polaritons compared to the condensate pump spots, see [31] for further information.

The phase difference between two coupled ballistically expanding condensates can be extracted from the resulting interference patterns in both real- and reciprocalspace. However, in the case of more complex geometries and couplings that may lead to frustration in the system, phase retrieval from intensity maps becomes less well defined, while at the same time, accuracy in the phase retrieval is important for the application of polariton lattices as simulators. In the following, we develop a homodyne interferometric technique that utilizes the $\mathrm{U}(1)$ symmetry in the classical phase configuration of coupled

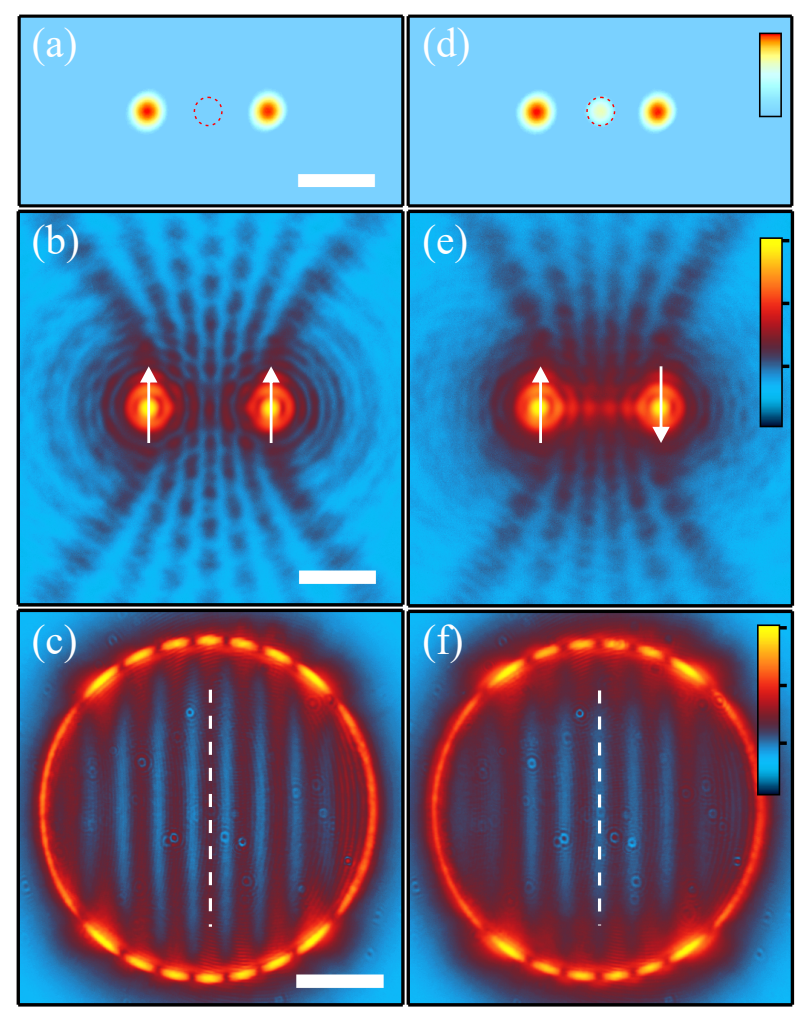

Figure 1. (a) Intensity profile of two non-resonant excitation spots used to imprint a polariton dyad in a FM configuration with corresponding normalized PL intensity shown in both (b) real- and (c) reciprocal-space. Introducing an optically-imprinted weak barrier in between the two condensates (d) switches the phase configuration of the dyad to an AFM state as shown (e,f) by the change in the interference pattern. White scale bars correspond to $10 \mu \mathrm{m}$, and $1 \mu \mathrm{m}^{-1}$ in real and reciprocal space respectively. Red dashed circles in $(\mathrm{a}, \mathrm{d})$ denote barrier position. White dashed vertical lines in $(\mathrm{c}, \mathrm{f})$ are guide to the eye. Colorscale in (a-f) is logarithmic and saturated at $7 \times 10^{-4}$ for (b), $1.25 \times 10^{-3}$ for $(c, e, f)$ and $2 \times 10^{-2}$ for $(\mathrm{a}, \mathrm{d})$.

polariton condensates, that allows for phase retrieval of all the condensates across a lattice.

The schematic in Fig. 2(a) shows the experimental configuration of the homodyne technique. An additional weak $\mathrm{CW}$ excitation laser $(\mathrm{FWHM} \approx 2 \mu \mathrm{m})$, resonant to the polariton condensate, is used to fix the phase of one condensate. The linewidth of the resonant excitation $(\approx$ $100 \mathrm{kHz}$ ) is more than five orders of magnitude narrower than that of the polariton condensate. Both pump and resonant seed are synchronously acousto-optically modulated. The emission of the whole polariton network is interfered with the resonant seed beam using a MachZehnder interferometer. This allows to extract the full phase map of the lattice with off-axis digital holography $[32,33]$. We apply this technique both to the polariton dyad (Fig. 1) and to the case of a single condensate, i.e. when one of the excitation pump spots of the dyad is blocked. The effect of the barrier onto the radial out- 


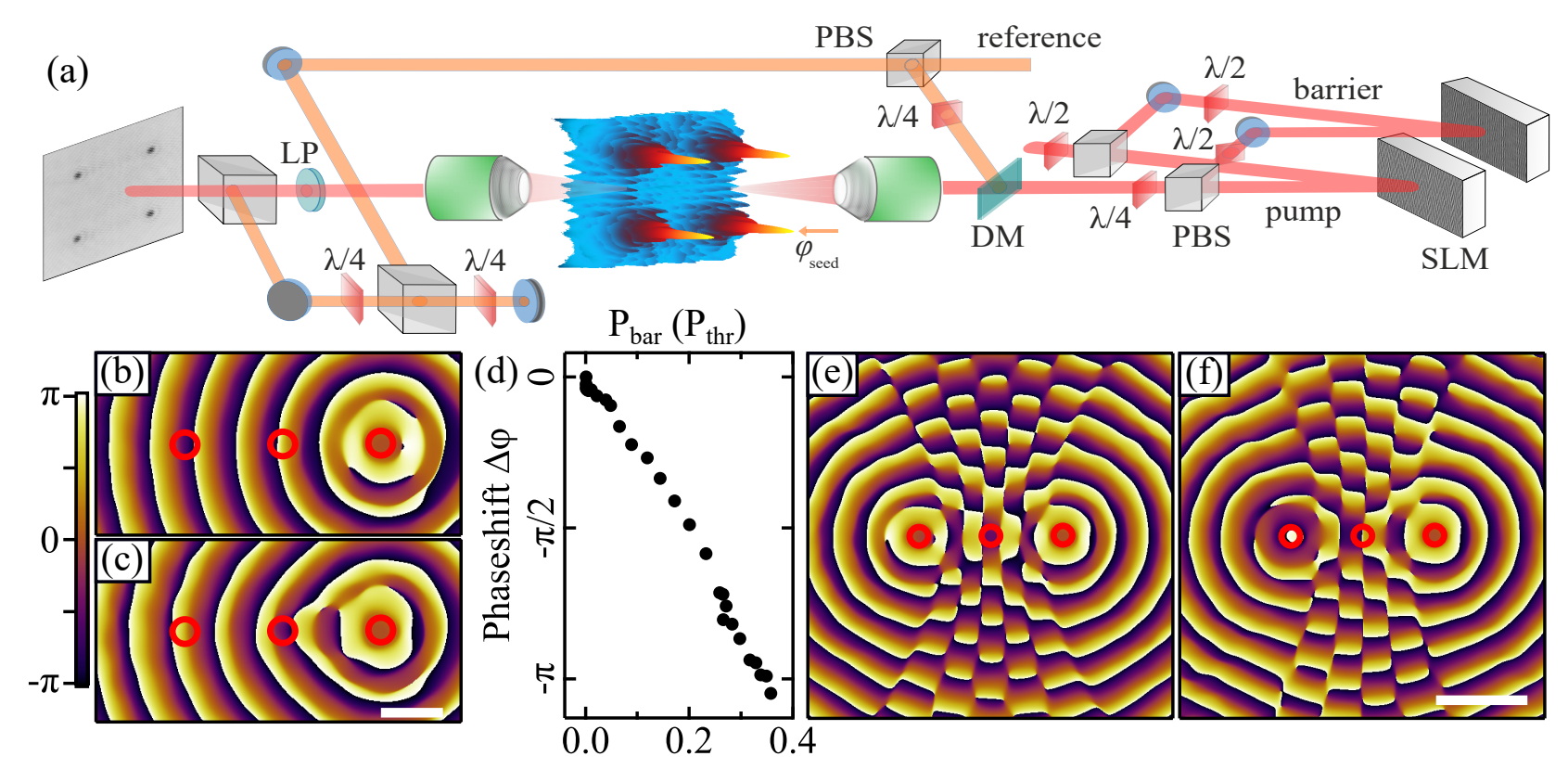

Figure 2. (a) Scheme of the setup that allows for imprinting of intercalated arrays of polariton condensates and potential barriers, through two separate SLMs, giving all-optical control of couplings. An additional weak laser beam (seed) resonant to the PL is coupled through a dichroic mirror (DM), onto one of the condensates fixing its phase and is subsequently used as a reference wave for interferometric homodyne measurements to implement phase readout. Measured phase maps of a single condensate (right-most red circle) pumped at $P=1.3 \times P_{\text {thr }}$ subject to a weak barrier (central red circle) with (b) $\mathrm{P}_{\mathrm{bar}}=0$ and (c) $\mathrm{P}_{\mathrm{bar}}=0.36 \times \mathrm{P}_{\mathrm{thr}}$. (d) The measured phaseshift $\Delta \varphi$ integrated within a circle of $1 \mu \mathrm{m}$ radius (left-most red-circle) at a position $d \approx 15.7 \mu \mathrm{m}$ away from the condensate shows a continuous decrease as a function of barrier pump power. The extracted phase maps (e,f) for the two configurations in Fig. 1 confirm the observed FM and AFM configurations by 0 and $\pi$ phase differences between the two condensate centres. The white scale bar in (c) [applying also to (b)] amounts for $5 \mu$ m, while the scale bar in (f) [applying also to (e)] amounts for $10 \mu \mathrm{m}$.

flow of polaritons from a single condensate can be seen in Fig. 2 for (b) $\mathrm{P}_{\mathrm{bar}}=0$ and (c) $\mathrm{P}_{\mathrm{bar}}=0.36 \times \mathrm{P}_{\mathrm{thr}}$. The red circles indicate, from right to left, the positions of a single pumped condensate, the barrier, and the mirrored location of the condensate with respect to the barrier. We subtract the obtained phase maps with and without the barrier, and extract the phase shift $\Delta \varphi$ of the condensate wavefunction at the location of the left most circle as a function of the barrier pump power. Figure 2(d) shows a continuous shift in $\Delta \varphi$ up to $-\pi$ up to the same barrier pump power that switches the parity of the dyad (Fig. 1). Figure 2(e,f) shows the obtained phase maps corresponding to the pumping profiles of Fig. 1(a,d). A similar dependency of the phaseshift under an optically generated potential was reported in Ref. [34] for polaritons propagating along a lithographic waveguide.

From the simplest configuration of the polariton dyad, we expand to a more complex system, the unit cell of a square lattice, wherein the coupling between condensates occurs not only with the nearest neighbors, but also with next nearest neighbors. Fig. 3(a) shows the pumping profile of the $2 \times 2$ polariton cell. The pump beams are co-circularly polarized and fixed at $1.34 \times \mathrm{P}_{\mathrm{thr}}$. The distribution of the polariton real-space PL [Fig. 3(b)] reveals AFM coupling between the four nodes, also corrob- orated by the reciprocal-space PL [Fig. 3(c)]. Here, the lattice constant $(\approx 21 \mu \mathrm{m})$ is chosen such that polariton condensation occurs at a single energy state.

We introduce a cross-circulary polarized pump in the center of the square that is kept at $0.5 \times \mathrm{P}_{\text {thr }}$ power [Fig. 3(e)]. From the interference pattern in both realand reciprocal-space PL we detect a switch from an AFM to a FM configuration [Fig. 3(f,g)]. This transition reveals that the central pump alters the coupling between next-nearest neighbor condensates (diagonal flows) dictating the resulting stable condensate phase configuration, see [31] for more information. Next, we add two additional barriers at the left- and right-edges of the cluster [Fig. 3(i)] to demonstrate control of nearest neighbor couplings. The intensity of the side barriers is $\approx 30 \%$ of the central barrier. Figure $3(\mathrm{j}, \mathrm{k})$ show the real- and reciprocal-space polariton PL in the presence of all three barriers. From the interference pattern we observe a switching from all FM to a paired ferromagnetic (PFM) coupling. This state is analogous to the compass state reported previously for both scalar [7, 35] and spinor polariton condensates [5]. We note that the transition between different phase ordered states is not digital but instead gradual as a function of barrier strength. Stationary phase configurations, such as presented in Fig. 1 and 
3, are separated by non-stationary (cyclical) states (as a function of barrier strength) which can be regarded as a superposition of the aforementioned stable configurations. This is in agreement with recent observations [19], where regimes of single-mode and multi-mode condensates were found depending on the pumping geometry. In [31] we show this transition between different phases (AFM, FM and PFM) with gradually increasing barrier heights. Corresponding extracted real space condensate phase maps are shown in Fig. $3(\mathrm{~d}, \mathrm{~h}, \mathrm{l})$.

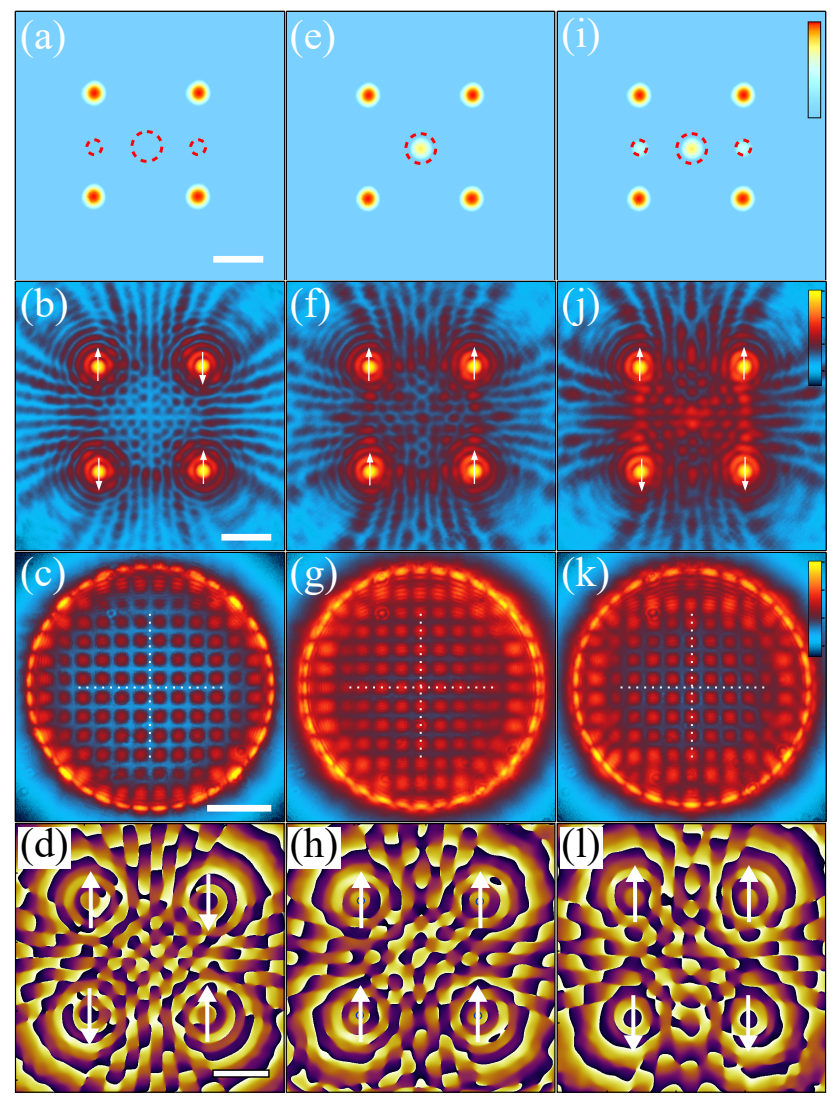

Figure 3. (a,e,i) Real-space intensity map of the non-resonant excitation geometry with barrier beams enclosed by red dashed circles. (b,f,j) Real- and $(\mathrm{c}, \mathrm{g}, \mathrm{k})$ reciprocal-space PL of the polariton condensates generated by the different barrier configurations (a,e,i) respectively showing AFM, FM, and PFM phases. Corresponding real-space phase maps are given in $(\mathrm{d}, \mathrm{h}, \mathrm{l})$ with white arrows denoting the magnetic arrangement of the cluster. Logarithmic colorscale in (i) applies to (a,e,i) and saturated at $2 \times 10^{-2}$, logarithmic colorscale in $(\mathrm{j})$ applies to $(\mathrm{b}, \mathrm{f}, \mathrm{j})$ and saturated at $3 \times 10^{-4}$, logarithmic colorscale in (k) applies to $(\mathrm{c}, \mathrm{g}, \mathrm{k})$ and saturated at $4.5 \times 10^{-4}$. The colorscale in $(\mathrm{d}, \mathrm{h}, \mathrm{l})$ is the same as in Fig. 2.

All experimental results are reproduced by numerical integration of the generalized Gross-Pitaevskii equation [31]. We also reproduce all observations using a recently developed discretized model describing the interacting expanding polariton condensates as time-delayed
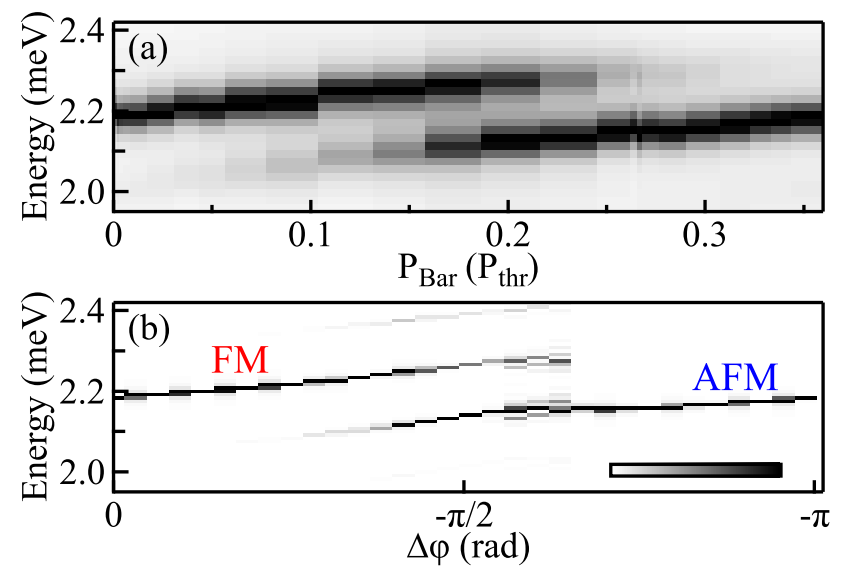

Figure 4. (a) Spectrally resolved PL of the polariton dyad (see Fig. 1) as a function of barrier pump power. (b) Numerically calculated spectra as a function of increasing coupling phase $\beta=\beta^{(0)}+\Delta \varphi$ obtained by integrating Eq.1 with random initial conditions. Energy is scaled with respect to the ground state of the lower polariton branch. Parameters: $d=15.7 \mu \mathrm{m}$, $\hbar \Omega=(1.45-i 0.5) \mathrm{meV}, \hbar \alpha=0.1 \mu \mathrm{eV}, \hbar R=0.5 \mu \mathrm{eV}, \hbar g=$ $0.5 \mu \mathrm{eV}, v=1.3 \mu \mathrm{m} \mathrm{ps}{ }^{-1}, P=100 \mathrm{ps}^{-1}, \Gamma_{R}=0.05 \mathrm{ps}^{-1}$, $\hbar J_{0}=1.1 \mathrm{meV}, k_{0}=(1.9+i 0.012) \mu \mathrm{m}^{-1}$ and $\beta^{(0)}=-0.8$ rad.

coupled oscillators [19], i.e.

$$
\begin{aligned}
i \dot{\psi}_{n} & =\left[\Omega+\left(g+i \frac{R}{2}\right) X_{n}+\alpha\left|\psi_{n}\right|^{2}\right] \psi_{n} \\
& +\sum_{m} J_{n m} e^{i \beta_{n m}} \psi_{m}\left(t-d_{n m} / v\right), \\
\dot{X}_{n} & =-\left(\Gamma_{R}+R\left|\psi_{n}\right|^{2}\right) X_{n}+P .
\end{aligned}
$$

Here $\psi_{n}$ represents the phase and amplitude of the $n$th condensate, $X_{n}$ their respective reservoirs, and the sum runs over the nearest and next-nearest neighbors. The complex self-energy of each condensate is captured in $\Omega$, the blueshift due to interactions and stimulated scattering rate (i.e., optical gain) from the reservoir is given by $g$ and $R$ respectively, $\alpha$ describes polariton-polariton interaction strength, $\Gamma_{R}$ the decay rate of the exciton reservoir, $P$ the non-resonant pump power, $J_{n m}$ quantifies the coupling strength between neighboring condensates, $\beta_{n m}$ describes the coupling phase, $v$ is the average phase velocity of polaritons outside their pump spots, and $d_{n m}$ is the distance between neighbors. We use similar parameters as in Ref. [19] with the exception of the coupling phase $\beta_{n m}$ which depends on the barrier strength according to Fig. $2(\mathrm{~b}-\mathrm{d})$, i.e. $\beta_{n m}=\beta_{n m}^{(0)}+\Delta \varphi$ with phase $\beta_{n m}^{(0)}$ in case of no barrier. For the case of the dyad given in Fig. 1, the spectrally resolved PL [see Fig. 4(a)] displays a gradual transition of the dyad mode from a FM to an AFM energy branch as a function of barrier strength, separated by a desynchronous domain, shown experimentally in [31], where both FM and AFM modes are populated. We find that the dominant effect of the 
barrier is to introduce a phase lag $\Delta \varphi$ to the transmitted condensate signal traveling to its nearest neighbor, which ultimately reverses the sign of the complex coupling when $\Delta \varphi=-\pi$. Numerically resolving the energies of Eq. (1) by varying the phase lag $\beta_{n m}$ indeed produces the same behavior in energy as seen in experiment [see Fig. 4(b)]. The phase lag is mutual in the dyad and we can write for brevity $\beta_{12}=\beta_{21}=\beta$. We conclude that optical control of the coupling phase with the barrier allows for control of both real and imaginary parts of the complex coupling between the nodes in networks of polariton condensates. This technique can be readily applied to extended lattices as shown in [31].

In conclusion, we have developed a new strategy of optically tuning the interactions between polariton condensates in a given two-dimensional network. This approach opens up the path to simulation of synchronization, periodic orbitals, and chaos in more complicated structures with desired nearest neighbor couplings. Moreover, we demonstrated a new experimental technique, which allows scanless readout of the condensates relative phases. Implementation of homodyne interferometry is advantageous for phase retrieval in cases where lattice geometry leads to non-trivial phase coupling between adjacent condensates.

Acknowledgements - The authors acknowledge the support of the Skoltech NGP Program (Skoltech-MIT joint project), and the UK's Engineering and Physical Sciences Research Council (grant EP/M025330/1 on Hybrid Polaritonics).

* Pavlos.Lagoudakis@soton.ac.uk

[1] D. Sanvitto and S. Kéna-Cohen, Nature Materials 15, 1061 EP (2016), review Article.

[2] T. Byrnes, K. Wen, and Y. Yamamoto, Physical Review A 85, 040306 (2012).

[3] Á. Cuevas, J. C. López Carreño, B. Silva, M. De Giorgi, D. G. Suárez-Forero, C. Sánchez Muñoz, A. Fieramosca, F. Cardano, L. Marrucci, V. Tasco, G. Biasiol, E. del Valle, L. Dominici, D. Ballarini, G. Gigli, P. Mataloni, F. P. Laussy, F. Sciarrino, and D. Sanvitto, Science Advances 4 (2018), 10.1126/sciadv.aao6814.

[4] D. D. Solnyshkov, A. V. Nalitov, and G. Malpuech, Phys. Rev. Lett. 116, 046402 (2016).

[5] H. Ohadi, A. J. Ramsay, H. Sigurdsson, Y. del ValleInclan Redondo, S. I. Tsintzos, Z. Hatzopoulos, T. C. H. Liew, I. A. Shelykh, Y. G. Rubo, P. G. Savvidis, and J. J. Baumberg, Phys. Rev. Lett. 119, 067401 (2017).

[6] S. Klembt, T. H. Harder, O. A. Egorov, K. Winkler, R. Ge, M. A. Bandres, M. Emmerling, L. Worschech, T. C. H. Liew, M. Segev, C. Schneider, and S. Höfling, Nature 562, 552 (2018).

[7] N. G. Berloff, M. Silva, K. Kalinin, A. Askitopoulos, J. D. Töpfer, P. Cilibrizzi, W. Langbein, and P. G. Lagoudakis, Nature Materials 16, 1120 (2017).

[8] C. Leyder, T. C. H. Liew, A. V. Kavokin, I. A. Shelykh,
M. Romanelli, J. P. Karr, E. Giacobino, and A. Bramati, Phys. Rev. Lett. 99, 196402 (2007).

[9] T. Gao, P. S. Eldridge, T. C. H. Liew, S. I. Tsintzos, G. Stavrinidis, G. Deligeorgis, Z. Hatzopoulos, and P. G. Savvidis, Phys. Rev. B 85, 235102 (2012).

[10] A. V. Zasedatelev, A. V. Baranikov, D. Urbonas, F. Scafirimuto, U. Scherf, T. Stöferle, R. F. Mahrt, and P. G. Lagoudakis, Nature Photonics , 1 (2019).

[11] A. Amo, T. C. H. Liew, C. Adrados, R. Houdré, E. Giacobino, A. V. Kavokin, and A. Bramati, Nature Photonics 4, 361 (2010).

[12] F. Marsault, H. S. Nguyen, D. Tanese, A. Lemaître, E. Galopin, I. Sagnes, A. Amo, and J. Bloch, Applied Physics Letters 107, 201115 (2015).

[13] H. S. Nguyen, D. Vishnevsky, C. Sturm, D. Tanese, D. Solnyshkov, E. Galopin, A. Lemaître, I. Sagnes, A. Amo, G. Malpuech, and J. Bloch, Phys. Rev. Lett. 110, 236601 (2013).

[14] I. Carusotto and C. Ciuti, Rev. Mod. Phys. 85, 299 (2013).

[15] H. Ohadi, Y. del Valle-Inclan Redondo, A. J. Ramsay, Z. Hatzopoulos, T. C. H. Liew, P. R. Eastham, P. G. Savvidis, and J. J. Baumberg, Phys. Rev. B 97, 195109 (2018).

[16] M. Abbarchi, A. Amo, V. G. Sala, D. D. Solnyshkov, H. Flayac, L. Ferrier, I. Sagnes, E. Galopin, A. Lemaître, G. Malpuech, and J. Bloch, Nature Physics 9, 275 (2013).

[17] K. Rayanov, B. L. Altshuler, Y. G. Rubo, and S. Flach, Phys. Rev. Lett. 114, 193901 (2015).

[18] M. Wouters, I. Carusotto, and C. Ciuti, Phys. Rev. B 77, 115340 (2008).

[19] J. D. Töpfer, H. Sigurdsson, L. Pickup, and P. G. Lagoudakis, Commun. Phys. 3, 2 (2020).

[20] H. Ohadi, R. L. Gregory, T. Freegarde, Y. G. Rubo, A. V. Kavokin, N. G. Berloff, and P. G. Lagoudakis, Phys. Rev. X 6, 031032 (2016).

[21] R. Lang and K. Kobayashi, IEEE Journal of Quantum Electronics 16, 347 (1980).

[22] A. Pikovsky, M. Rosenblum, J. Kurths, and J. Kurths, Synchronization: a universal concept in nonlinear sciences, Vol. 12 (Cambridge university press, 2003).

[23] A. Marandi, Z. Wang, K. Takata, R. L. Byer, and Y. Yamamoto, Nature Photonics 8, 937 (2014).

[24] P. L. McMahon, A. Marandi, Y. Haribara, R. Hamerly, C. Langrock, S. Tamate, T. Inagaki, H. Takesue, S. Utsunomiya, K. Aihara, R. L. Byer, M. M. Fejer, H. Mabuchi, and Y. Yamamoto, Science 354, 614 (2016).

[25] F. Barahona, Journal of Physics A: Mathematical and General 15, 3241 (1982).

[26] K. P. Kalinin and N. G. Berloff, Advanced Quantum Technologies 3, 1900065 (2020).

[27] C. Schneider, K. Winkler, M. D. Fraser, M. Kamp, Y. Yamamoto, E. A. Ostrovskaya, and S. Höfling, Reports on Progress in Physics 80, 016503 (2016).

[28] A. Amo, S. Pigeon, C. Adrados, R. Houdré, E. Giacobino, C. Ciuti, and A. Bramati, Phys. Rev. B 82, 081301 (2010).

[29] A. Askitopoulos, H. Ohadi, A. V. Kavokin, Z. Hatzopoulos, P. G. Savvidis, and P. G. Lagoudakis, Phys. Rev. B 88, 041308 (2013).

[30] P. Cilibrizzi, A. Askitopoulos, M. Silva, F. Bastiman, E. Clarke, J. M. Zajac, W. Langbein, and P. G. Lagoudakis, Applied Physics Letters 105, 191118 (2014). 
[31] See Supplemental Material [url] for details on numerical simulations and results on couplings control in polariton clusters, which includes Refs. [36, 37].

[32] T. Kreis, J. Opt. Soc. Am. A 3, 847 (1986).

[33] M. Liebling, T. Blu, and M. Unser, J. Opt. Soc. Am. A 21, 367 (2004).

[34] C. Sturm, D. Tanese, H. Nguyen, H. Flayac, E. Galopin, A. Lemaître, I. Sagnes, D. Solnyshkov, A. Amo, G. Malpuech, et al., Nature communications 5, 3278 (2014).
[35] E. Z. Tan, H. Sigurdsson, and T. C. H. Liew, Phys. Rev. B 97, 075305 (2018).

[36] M. Wouters and I. Carusotto, Phys. Rev. Lett. 99, 140402 (2007).

[37] Y. del Valle-Inclan Redondo, H. Ohadi, Y. G. Rubo, O. Beer, A. J. Ramsay, S. I. Tsintzos, Z. Hatzopoulos, P. G. Savvidis, and J. J. Baumberg, New J. Phys. 20, 075008 (2018). 